\title{
Anthologie musicale de la péninsule arabique. Collection
} Simon Jargy.

Archives Internationales de Musique Populaire, 1994

\section{Scheherazade Qassim Hassan}

\section{(2) OpenEdition}

\section{Journals}

Édition électronique

URL : http://journals.openedition.org/ethnomusicologie/940

ISSN : 2235-7688

\section{Éditeur}

ADEM - Ateliers d'ethnomusicologie

\section{Édition imprimée}

Date de publication : 1 décembre 1997

Pagination : 348-354

ISBN : 2-8257-0579-9

ISSN : 1662-372X

\section{Référence électronique}

Scheherazade Qassim Hassan, "Anthologie musicale de la péninsule arabique. Collection Simon Jargy. », Cahiers d'ethnomusicologie [En ligne], 10 | 1997, mis en ligne le 06 janvier 2012, consulté le 10 décembre 2020. URL : http://journals.openedition.org/ethnomusicologie/940

Ce document a été généré automatiquement le 10 décembre 2020.

Tous droits réservés 


\title{
Anthologie musicale de la péninsule arabique. Collection Simon Jargy.
}

\author{
Archives Internationales de Musique Populaire, 1994
}

Scheherazade Qassim Hassan

\section{RÉFÉRENCE}

Anthologie musicale de la péninsule arabique. Collection Simon Jargy. vol.1: Poésie chantée des Bédouins ; vol. 2 : Musique des pêcheurs de perles; vol. 3 : Le sowt, musique des villes; vol. 4 : Le chant des femmes. Notices bilingues (français-anglais) de Simon Jargy et MarieGeorges Caussade-Jargy. Un coffret de 4 CD AIMP (Archives Internationales de Musique Populaire) XXX-XXXIII / VDE 780-783, 1994.

1 Il est temps que l'histoire écrite de la musique arabe, de ses deux écoles connues du Maghreb et du Mashrek, se complète et s'enrichisse des nouvelles découvertes musicales et musicologiques relativement récentes qui dévoilent la très grande richesse et la diversité musicale dans la Péninsule arabique. L'expérience récente du terrain d'une des traditions les plus anciennes des Arabes et de leurs rencontres depuis toujours avec les peuples voisins met à notre disposition des éléments qui élargiront la définition courante de cette musique et permettront une compréhension et une analyse plus fiable. Désormais, grâce à une connaissance concrète des divers éléments de la musique de la Péninsule, on peut plus aisément parler en termes comparatifs de cette musique et de celles des autres régions arabes.

2 Le professeur Simon Jargy nous propose quatre $\mathrm{CD}$ réunis sous le titre "Anthologie musicale de la Péninsule Arabique»: vol.1: Poésie chantée des Bédouins; vol.2: Musique des pêcheurs de perles; vol. 3 : Le sowt, musique des villes; vol. 4 : Le chant des femmes. Tous les exemples musicaux proviennent de la collection personnelle de l'auteur mais ne sont pas exclusivement ses propres enregistrements. En fait, plusieurs chercheurs et institutions ont contribué à cette collecte. Parmi eux figurent le regretté Poul Rovsing Olsen et Ali al-Ansari du Koweit. Mohammed Ghanim du Yémen propose des 
enregistrements anciens de sa propre collection et le Centre des musiques traditionnelles d'Oman y participe également avec quelques pièces.

3 Ainsi, parmi les grandes traditions communes de la Péninsule, seule la musique afroarabe est absente. On aurait aimé qu'elle complète cette anthologie. Le quatrième volume, "Le chant des femmes" représente un choix de chants de mariage - ou de divertissement-, de travail et quelques chants spécifiques du répertoire féminin.

On peut regretter que tous les pays de la Péninsule ne soient pas représentés : la plupart des exemples proviennent du Bahrein (quarante plages), huit d'Abu Dhabi, six du Koweit, deux d'Oman, sept du Yémen et trois autres de la Syrie en dehors de la Péninsule. Quant à l'Arabie Saoudite et le Qatar, ils sont absents.

\section{Vol. 1 : Poésie chantée des Bédouins}

5 Seize exemples musicaux constituent le disque I de cette anthologie consacrée à la tradition bédouine. Ils proviennent seulement de deux pays de la Péninsule : le Koweit , avec cinq exemples musicaux , et l'Emirat d'Abu Dhabi, un des sept Emirats faisant partie des Emirats Arabes Unis, avec sept exemples. Les cinq autres pays de la Péninsule; l'Arabie Saoudite, le Yémen, Oman, le Qatar et le Bahrein sont absents de ce volume. Ces traditions bédouines de base s'étendent, au delà de la Péninsule jusqu'en Irak, en Syrie, au Liban et en Palestine, où elles trouvent leurs équivalents et leurs prolongements. Cependant, choisir dans ce disque consacré à la Peninsule, de ne représenter que la Syrie - avec trois exemples - parmi tous les pays du Proche-Orient, est logiquement peu justifié, quoique intéressant : l'Irak et la Jordanie aurtaient aussi dû y figurer.

6 L'ensemble des pièces du volume a été enregistré par Simon Jargy et Poul Rovsing Olsen entre 1970 et 1972 ; on aurait aimé voir devant chaque exemple le nom de son collecteur. Aussi on ne comprend pas très bien pourquoi Jargy, en parlant de ses enregistrements, indique que le musicologue koweitien Ahmed Ali avait mis à sa disposition quelques enregistrements, alors qu'on ne les trouve pas dans ce disque.

7 La musique bédouine a fait l'objet de très peu de recherches de la part des ethnomusicologues, alors qu'elle subsiste toujours dans les régions mentionnées. Elle est fondée sur le personnage du poète (shaer),qui doit être capable de dire instantanément la poésie, de la chanter, tout en s'accompagnant à la vièle monocorde rabab. Dans cette tradition, réciter la poésie et la chanter se fondent en un seul terme, anshada, « récitation » ou cantillation. Aujourd'hui encore, dans les pays où la tradition bédouine est présente, les poètes chantent leurs propres créations poétiques pour vérifier la justesse des accents métriques, brefs et longs. L'essentiel dans le chant bédouin est le verbe et le sens qui s'en dégage. Ainsi le mot "chanteur ", inexistant dans la tradition bédouine, est remplacé par shaer. La musique elle-même est limitée dans son ambitus à une quarte ou une quinte, avec des phrases musicales généralement courtes qui correspondent aux mètres poétiques. On assiste dans les différentes formes musicales bédouines, à une répétition - avec variations - de la même phrase mélodique. Contrairement aux formules rythmiques de la musique citadine, on est confronté ici à des rythmes binaires ou ternaires courts et repétitifs. Les exemples de musique bédouine figurant sur ce $\mathrm{CD}$ correspondent à la musique des Bédouins nomades du Proche Orient, alors que d'autres éléments caractérisent celle des Bédouins semi-sédentaires et celle des Bédouins sédentarisés. 
8 Ici, les chants bédouins peuvent être groupés, selon l'interprète et la fonction du chant, en trois catégories: 1 . Le chant soliste, qui représente l'essentiel du répertoire bédouin par la diversité de ses formes et l'importance donnée au poème (onze plages sur seize). 2. Le chant avec alternance d'un soliste et d'un groupe, toujours lié à la danse collective et à des circonstances solennelles,.. qu'elles soient patriotiques ou relatives au cycle de la vie et au divertissement (plage 8). 3. Les chants antiphoniques d'origine guerrière interprétés par deux chœurs qui pratiquent également la danse (plages 11, 12 et 13). Un seul exemple représente le solo de rabab.

9 Le plus grand problème qui apparait pour le moment, dans les recherches sur la musique bédouine est tout ce qui touche au domaine de la terminologie et de la définition. Mettre de l'ordre dans cette confusion reste un but à réaliser. Simon Jargy nous met en garde : « Les appellations données à l'un ou l'autre genre restent imprécises et désignent tantôt la forme poétique (maqal) ou le thème (ghazal), tantôt le rythme prosodique ou musical ( shkiri, samri, 'arda, harbiyya, etc.) ». Un lecteur non-averti pourrait être complètement désorienté s'il ne suivait que les exemples musicaux et les commentaires du texte. En effet, l'aspect poétique, l'aspect musical et l'aspect fonctionnel d'un même chant peuvent être désignés par plusieurs termes. Inversement, un seul terme ne peut donner une idée complète du type du chant auquel il se réfère.

10 Pour commencer et pour éviter toute équivoque sur l'emploi du terme nabati qui apparaît à plusieurs reprises dans le texte, il convient de préciser que nabati s'applique au seul aspect poétique et désigne la poésie populaire et vernaculaire de la Péninsule Arabique, en opposition avec la poésie écrite en arabe classique. Par conséquent, toute la poésie chantée dans ce disque appartient au dialecte nabati. Selon le livret du CD, plusieurs chants, bien qu'ils portent des noms différents sont des qisid. Ainsi, le mashub (plage 1) maqal (plage 5), mangus (plage 6), nawhi (plage 8) et skhiri (plages 9 et 10) appartiennent au gisid. Un ghazal, terme qui fait reférence au contenu poétique, pourrait être aussi un qasid. Le terme samri, seul ou ajouté à un autre signifie l'aspect rythmique de la danse. Si le skhiri (plage 9) est du genre qasid non mesuré, le skhiri (plage 10) est du genre samri rythmé qui se prête à la danse. Les thèmes de la musique bédouine ne sont pas réservés à un genre. A part le ghazal, qui se réfère de toute façon au contenu amoureux, les differents genres peuvent comporter des panégyriques, des chants d'exaltation, de nostalgie, d'amour ou de combat guerrier.

\section{Vol. 2 : Musique des pêcheurs de perles}

11 Connu dans les pays du Golfe sous les noms de fann al bahar, «l'art de la mer », d'aghani al ghaws, «chants de plongée», ou encore comme ici de nahma, il s'agit d'un grand répertoire, encore non totalement inventorié par les ethnomusicologues, et constitué de nombreux chants interprétés par les pêcheurs de perles d'une région qui s'étend entre le Koweit et Oman. Ces chants étaient jadis de deux sortes : liés aux activités même de la pêche de perles ou interprétés après les heures du travail ou après la fin de la saison de pêche. Dans ce dernier cas, on voit apparaître des instruments de musique, des claquements de mains, voire des danses. Depuis plus de deux décennies, avec la diminution et la disparition de l'économie de la pêche, concurrencée à la fois par les perles de culture japonaises et par l'apparition du pétrole, ces chants sont pratiqués hors contexte par les très nombreux ensembles professionnels (firaq), constitués au départ par 
les vieux plongeurs et marins installés dans al-dour al-sha'biyya, les «maisons populaires », et qui chantent à la demande dans tous les pays du Golfe.

Découvert très tardivement et encore peu connu tant des Arabes que du monde des spécialistes, «l'art de la mer » est sans doute millénaire puisque les perles de la région étaient connues déjà des Sumériens. Considéré comme un répertoire populaire, il est non codifié et n'est pas basé sur une théorie connue. Il représente un des aspects les plus intéressants et émouvants de la musique arabe avec une originalité très particulière.

Dans le présent CD nous trouvons trois cycles de "l'art de la mer », nommé ici nahma, provenant de Bahrein et interpretés par le même ensemble de dar Jina'. Les cycles 1 et 2 furent enregistrés par Simon Jargy en 1970 et le cycle 3 a été enregistré en 1975 en collaboration avec Ali al-Ansari. Il faut rappeler que beaucoup d'autres ensembles et d'autres "traditions de la mer", voire d'autres variantes continuent à exister dans les différentes villes du Golfe.

Jargy nous dit que « les origines de ces formes et structures doivent être recherchées dans les traditions autres que proprement arabes ». A ma connaissance, outre la musique des Bédouins nomades, toutes les musiques de la région sont faites de rencontres et d'assimilation, au point qu'il est souvent très difficile d'en établir les origines. Cette antique et étonnante musique est, depuis toujours, liée à l'activité de la pêche de perles qui concernait les riverains du Golfe et ceux qui s'y s'installèrent pour gagner leur pain dans ce domaine. Depuis des siècles, elle est réservée a ceux qui travaillent dans la pêche, quelle que soit leur origine ou leur appartenance géographique. C'est un art issu en quelque sorte de "l'internationale des pêcheurs de perles", basé sur les rencontres, depuis des millénaires, entre Arabes, arabisés d'origine africaine et indo-iraniens. Le produit final est un genre à part qui ne ressemble qu'à lui-même. Néamoins, il est chanté en langue arabe, basé sur des formes poétique arabes (avec des références dans ce CD à des villes arabes comme Bagdad ou Alep), interprété en cycles qui nous rappellent les cycles classiques du maqâm irakien ou la wasla d'Alep, dans lesquels se succèdent des formes variées et où soliste et chœur alternent ou se superposent. Par son aspect polyphonique, où se superposent deux à trois voix ponctuées par les bruits forts de l'expiration, il rappelle le dhikr soufi tel qu'il est pratiqué au Proche Orient. C'est dans son aspect rythmique ou polyrythmique complexe et par cet impressionnant bourdon unique en son genre que l'art de la mer est original. Au plan terminologique, on constate des similitudes avec l'Irak. Le terme tanzila, qui est ici une invocation religieuse, est aussi connu dans la ville de Mossoul où il désigne le muwashah religieux. Le terme zheiri ,d'origine irakienne, dérivé du nom d'un auteur de mawwal, correspond en Irak uniquement aux mawwal sapiental et non pas aux autres types de structures poétiques de mawwal invoqués dans le texte.

Dans les trois cycles de nahma proposés par Simon Jargy, on peut retrouver les éléments qui constituent le langage de cet art : le chant mélismatique du soliste (nahham); le chant choral, parfois responsorial; le bourdon grave; les cycles rythmiques complexes avec beaucoup d'irrégularités conjuguées à la pratique de contre-accents; l'usage des instruments de percussion (membranophones et idiophones) ; les claquements de mains ; les bruits et l'expiration bruyante liés à l'origine aux gestes du travail, l'utilisation de poèmes de types mawwal et de syllabes hors texte. Mais tous ces éléments ne se retrouvent pas obligatoirement dans chaque chant. Ainsi, dans le très beau cycle nahma 3 $\mathrm{du} C D$, le bourdon grave est absent de tous les chants constituant le cycle. Et dans le quatrième chant de ce cycle khatfat al-shira' - à l'origine un chant de travail - seul le 
chœur apparaît avec les claquements de mains et les rythmes. D'autres combinaisons sont possibles.

Plusieurs questions restent à poser : y a -t-il aujourd'hui un nombre connu de cycles ? y at-il des cycles fixes quant à l'ordre des chants les constituant? Apparemment, les trois cycles proposés sont des cycles reconstitués en toute liberté avec des éléments récurrents dans les autres cycles. Les changements apparaissent, comme dans les autres musiques du Proche Orient, sur le plan textuel et par une forme d'interprétation libre. D'autres chants - ici les chants de travail - diffèrent dans chacun des trois cycles. On peut imaginer qu'à l'instar des cycles musicaux contemporains et reconstitués du Proche Orient, une certaine liberté permet d'inclure ou d'exclure les différents chants. Reste à savoir s'il y avait un nombre connu des cycles lorsque ces chants se pratiquaient dans le contexte de mer, si aujourd'hui les différents ensembles firaq du Golfe représentent quelques modèles de cycles, ou si la liberté absolue est la règle.

\section{Vol. 3 : Le sowt, musique des villes}

Le sowt est un des principaux genres de la musique citadine dans la Péninsule. De langue classique, semi populaire, voire populaire, le sowt se fonde sur des structures mélodicorythmiques d'une grande complexité et se présente sous forme de cycles accompagnés au luth oriental adopté au XIX ${ }^{\mathrm{e}}$ siècle, qui a remplacé le vieux luth de la Péninsule al-qanbus.

Le sowt est aussi un des plus anciens genres de la musique arabe ; il est cité dans l'ouvrage d'Abou-l-Farag al Isbahani ( $\mathrm{X}^{\mathrm{e}}$ siècle) décrivant les cent sawt choisis à Bagdad, dignes d'être présentés devant la Caliphe Haroun al-Rashid. Dans un deuxième temps, on retrouve le sowt comme un art illustre des villes du Yémen, ainsi qu'au sein de la communauté yéménite résidente en Inde, conservatrice et grande gardienne de l'ancienne tradition yéménite ; et il y a encore le sowt de l'archipel historique de Bahrein. Au Koweit la tradition du sowt s'instaure avec Abdallah al-Faraj (1836-1903), qui vécut à proximité de la communauté yéménite de l'Inde et à qui les légendes populaires attribuent la paternité, la découverte, voire l'invention du genre au Koweit. L'inspiration des rythmes indiens attribués à al-Faraj n'est pas une originalité personnelle. Partout dans le Golfe, cette fascination apparaît d'une manière très assimilée au point que les Indiens ne s'y reconnaissent pas.

Le volume comporte onze exemples de sowt d'appartenances diverses : al-sowt al-shami (le sowt de la Syrie ou de Damas, trois plages), al-sowt al-'arabi (le sowt arabe, une plage), alsowt al-bahraini (le sowt de Bahrein, une plage) ; al-sowt al-san'ani (le sowt de San'a, Yémen, trois plages), ainsi que deux chansons n'appartenant pas au genre même, mais qui sont d'habitude interprétées à la fin du cycle. Rien dans le texte ne nous permet de comprendre à quoi correspondent ces differents types de sowt. Certes, il s'agit d'un problème très épineux qui n'a pas été suffisamment clarifié dans les écrits arabes. Dans un cycle de sowt,l'improvisation instrumentale est suivie par une introduction vocale non rythmée (tahrira), suivie par le sowt proprement dit qui se caractérise par l'accompagnement d'une formule rythmique claire. A la fin du cycle apparait la tawshiha qui est d'habitude suivie d'une chanson (pasta). Des considérations complexes concernant le mètre, le type reponsoriel qui s'établit entre le soliste et le chœur, le refrain, le poème qui se prête ou non à la déclamation, le type du rythmique, permettent aux spécialistes d'établir les lignes de démarcation entre les différents sowt. Mais leurs critères de 
distinction ne sont pas forcement opératoires. En tout état de cause,on n'a à l'écoute pas la moindre difficulté à constater l'affinité de tous les sowt ici présents avec ce qu'on connaît du genreyéménite .

\section{Vol. 4 : Le chant de femmes} les pays du Golfe qu'on arrive à avoir le plus d'exemples de musique de femmes grâce à la tradition répandue des groupes ou ensembles (firaq) professionnels masculins et féminins qui se produisent dans toutes les circonstances de la vie sociale. Aucun interdit ne pèse sur l'apparition publique de ces groupes de femmes, et les différentes télévisions locales transmettent regulièrement des programmes qui montrent des ensembles féminins connus. de ces exemples proviennent de Bahrein et sont interprétés par quatres ensemblesdifférents constitués exclusivement de femmes. En revanche, dans les deux exemples de chant omanais, qui proviennent de la collection du Centre de musique traditionnelle de Mascate, l'ensemble est mixte et l'élément féminin se limite à la chanteuse soliste, alors que les percussionnistes sont des hommes. Les trois exemples du Yémen sont interpréts par des fillettes et ne sont pas d'une grande représentativité des chants de femmes yémenites.

Il est peut-être utile de rappeller que, hormis le Yémen et Oman, les chants présentés ici comme étant de Bahrein représentent en fait un répertoire commun aux pays riverains du Golfe. Des versions koweitiennes, qataris ou provenant des Emirats sont aussi disponibles. des fillettes, bien que très limités musicalement, sont représentatifs d'un thème qui touchait la vie de toute la population, à savoir, la pêche de perles. Et il y a tout un répertoire de femmes qui aborde cette activité par le biais des imprécations typiques contre la mer et ses dangers, contre le propriétaire du bateau, terrible exploitant des hommes. A cela se conjugue, avec une grande force émouvante, l'expression d'un tendre amour à tous ces hommes dont le retour est attendu avec impatience et angoisse.

L'ensemble des chants présentés sous le titre « chant de mariage » sont en fait des chants de divertissement et de réjouissances afrah, non exclusifs aux mariages. Seuls les mrada 1 et 2 touchent directement au sujet du mariage. Ces chants représentent deux types d'interprétations responsorielles. Aux chants regroupés sous le titre de chants de mariage, peuvent s'ajouter d'autres chants appartenant aux mêmes catégories de divertissements représentant des genres musicaux rythmés qui portent des noms différents, mais invitant tous à la danse, donc appartenant au groupe samri. Il est dommage que certains enregistrements, y compris ceux provenant d'Oman, ne soient pas de très bonne qualité sonore et que la voix soliste soit lointaine et couverte par la percussion et le chœur. Ceux du Yémen représentent l'apprentissage à l'école coranique et les chants des travaux agricoles.

L'ensemble de tous les chants réunis sur ces quatres CD étonne par la récurrence si fréquente des diverses formes responsoriales et par l'alternance entre le chant soliste et le chant du chœur, si importante dans l'interprétation vocale de la région. Le Golfe serait-

Cahiers d'ethnomusicologie, 10 | 1997 
il cette région du monde arabe qui propose un équilibre entre le rôle de l'individu et le rôle de la communauté? 